\title{
Fatores de risco para retinopatia diabética
}

\author{
Risk factors for diabetic retinopathy
}

\author{
Maria Cristina Boelter ${ }^{1}$ \\ Mirela Jobim de Azevedo ${ }^{2}$ \\ Jorge Luiz Gross ${ }^{3}$ \\ Jacó Lavinsky ${ }^{4}$
}

\begin{tabular}{|l|}
\hline \multicolumn{2}{|c|}{ RESUMO } \\
\hline A retinopatia diabética (RD) é uma complicação crônica do diabete melito \\
(DM) que, após 20 anos de duração de diabete melito, ocorre em $99 \%$ dos \\
pacientes com diabete melito tipo 1 e em $60 \%$ dos pacientes com diabete \\
melito tipo 2, sendo a principal causa de cegueira em adultos. Níveis de \\
glicose e pressão arterial elevados, junto com longo tempo de duração do \\
DM, são os principais fatores de risco. Os fatores de risco para retinopatia \\
diabética podem ser classificados como genéticos e não genéticos, onde \\
estão incluídos aqueles relacionados ou não ao diabete melito, ambientais \\
e oculares. O controle dos fatores de risco conhecidos e o tratamento \\
adequado são a principal base do manejo da retinopatia diabética. O \\
objetivo desta revisão é fornecer ao oftalmologista uma informação atua- \\
lizada destes fatores, com ênfase no aspecto preventivo de perdas visuais \\
no paciente com diabete melito.
\end{tabular}

Descritores: Diabetes mellitus/complicações; Retinopatia diabética/etiologia; Fatores de risco; Teste de tolerância a glucose; Hipertensão; Nefropatia diabética/etiologia; Baixa visão

\section{INTRODUCÃ̃O}

O diabete melito (DM) é uma síndrome metabólica complexa em que ocorre uma deficiência relativa ou absoluta de insulina afetando o metabolismo dos carboidratos, lipídios e proteínas. Cerca 85 a $90 \%$ dos pacientes portadores de DM possuem o chamado DM tipo 2, previamente denominado DM não dependente de insulina ${ }^{(1)}$. Estima-se que no Brasil 7,6\% da população urbana entre 30 e 69 anos apresente DM, sendo que $46 \%$ dos casos não se sabem portadores de $\mathrm{DM}^{(2)}$. O DM está associado a complicações crônicas cardiovasculares, do sistema nervoso periférico e autonômico e microvasculares. Uma das complicações microvasculares mais importantes do DM é a retinopatia diabética (RD), que é a principal causa de novos casos de cegueira entre norte-americanos nas idades de 20 a 64 anos, causando 8000 novos casos de cegueira a cada ano ${ }^{(3)}$. No Brasil, estima-se que metade dos pacientes portadores de DM seja afetada pela RD, sendo responsável por 7,5\% das causas de incapacidade de adultos para o traba1 ho ${ }^{(4)}$ e por $4,58 \%$ das deficiências visuais ${ }^{(5)}$.

A hipóxia tecidual, acompanhada da perda da auto-regulação dos vasos retinianos, é o fator desencadeante da RD. Entretanto, a patogênese da doença microvascular retiniana não está totalmente esclarecida. A hiperglicemia está associada a outros possíveis fatores causais como dano celular mediado por alterações no metabolismo da aldose-redutase, fatores vasoproliferativos produzidos pela retina ("vascular endothelial growth factor"VEGF), hormônio de crescimento, anormalidades eritrocitárias, plaquetárias e na viscosidade sangüínea ${ }^{(6)}$.

A RD tem início nos capilares retinianos pelo comprometimento da barreira hemato-retiniana ${ }^{(7-8)}$. As alterações fundoscópicas seguem um curso 
progressivo, desde a RD leve, caracterizada por aumento da permeabilidade vascular, até a moderada e a grave, caracterizada por oclusão vascular e conseqüente proliferação fibrovascular (neovasos na retina e face posterior do vítreo) e cicatrização $^{(9)}$. A classificação da RD, adotada pela Academia Americana de Oftalmologia ${ }^{(10)}$ considera a presença destes estágios evolutivos, conforme está descrito no Quadro 1. Em qualquer estágio da RD pode ocorrer diminuição importante da acuidade visual causada por edema macular. Edema macular é definido pela presença de espessamento retiniano por acúmulo de fluidos dentro de 150 a $300 \mu$ do centro da mácula, e é a maior causa de diminuição da acuidade visual, principalmente em pacientes com DM tipo $2^{(3)}$.

A cegueira está associada à fase avançada da RD representada pela RD proliferativa e suas manifestações: hemorragia pré-retiniana ou vítrea, proliferação fibrovascular e descolamento tracional de retina, neovasos de íris e glaucoma neo$\operatorname{vascular}^{(3)}$. Quanto mais grave o estágio da RD, maior é o risco de surgimento de redução grave de visão, sendo a incidência cumulativa em 8,4 anos cerca de $2 \%^{(11)}$. Além disso, a incidência de RD proliferativa é aproximadamente o dobro em pacientes com DM tipo 1 quando comparados a pacientes com DM tipo $2^{(11)}$. A proporção de pacientes com RD aumenta progressivamente com a duração de DM, sendo que a maioria dos pacientes, em especial pacientes com DM tipo 1, vão apresentar algum grau de RD após cerca de 20 anos de duração de DM. Entretanto, considerando a mesma duração de DM, a proporção de pacientes com RD proliferativa varia de $40 \%$ a $60 \%{ }^{(12-15)}$. Esta diferença na incidência de RD proliferativa e outros estágios de RD sugere a existência de mecanismos patogênicos e fatores de risco próprios para a forma proliferativa da doença.

Não existe cura para a RD, estando os esforços terapêuticos concentrados nos fatores de risco para o aparecimento e agravamento da doença retiniana e no tratamento cirúrgico das lesões com alto risco de evolução para perda visual. Os estudos multicêntricos que vêm sendo realizados nos últimos trinta anos (DRS - "Diabetes Retinopaty Study"; ETDRS "Early Treatment Diabetic Retinopathy Study"; DRVS"Diabetic Retinopathy Vitrectomy Study"; DCCT -"Diabetes Control and Complications Study"; WESRD -“Wisconsin Epidemiologic Study of Diabetic Retinopathy"; UKPDS-'United Kingdom Prospective Diabetic Study") permitiram o estabelecimento de recomendações quanto ao acompanhamento e manejo da RD, além de reforçar a importância dos fatores de risco associados à RD.

O objetivo deste trabalho é revisar os principais fatores de risco relacionados ao desenvolvimento da $\mathrm{RD}$, em especial da RD proliferativa, cujo controle é de extrema importância para diminuir a progressão para a perda visual. Os estudos sobre fatores associados à RD avaliam prevalência e/ou incidência e/ou progressão de RD em geral, da RD proliferativa, da maculopatia e da redução ou perda visual.

\section{FATORES DE RISCO PARA RETINOPATIA DIABÉTICA}

Os fatores de risco podem ser classificados como genéticos e não genéticos. Entre os fatores não genéticos estão aqueles relacionados à presença do DM, como duração do DM, controle glicêmico e nefropatia diabética, e fatores não relacionados diretamente ao DM, como a hipertensão arterial sistêmica (HAS), dislipidemia, fatores ambientais e fatores oculares.

\section{Fatores de risco não genéticos}

\subsection{Fatores de risco relacionados diretamente ao DM}

\section{Duração do DM}

A duração do DM está fortemente associada com a freqüência e severidade da $\mathrm{RD}^{(16-19)}$. Após 20 anos de duração de $\mathrm{DM}$ quase todos os pacientes com DM tipo 1 e mais do que $60 \%$ dos pacientes com DM tipo 2 apresentam algum grau de RD, sendo $20 \%$ do tipo proliferativo ${ }^{(13-14,20-22)}$. Até $21 \%$ dos pacientes porta-

\begin{tabular}{|c|c|}
\hline \multicolumn{2}{|c|}{ Quadro 1. Classificação da retinopatia diabética } \\
\hline Classificação & Características fundoscópicas \\
\hline \multicolumn{2}{|c|}{ Retinopatia diabética não proliferativa } \\
\hline Mínima & Raros microaneurismas \\
\hline Leve & Poucas hemorragias dispersas e microaneurismas \\
\hline Moderada & $\begin{array}{l}\text { Moderadas hemorragias e microaneurismas, exudatos duros e algodonosos podem } \\
\text { estar presentes. } \\
\text { Hemorragias intra-retinianas em todos os quadrantes. }\end{array}$ \\
\hline Grave ou muito grave & $\begin{array}{l}\text { Veias em rosário em pelo menos dois quadrantes. } \\
\text { Anormalidades microvasculares intra-retinianas em pelo menos } 1 \text { quadrante. }\end{array}$ \\
\hline \multicolumn{2}{|c|}{ Retinopatia diabética proliferativa } \\
\hline Baixo risco & $\begin{array}{l}\text { Neovascularização retiniana ou do disco óptico, mas sem atingir caracte- rísticas de alto } \\
\text { risco. }\end{array}$ \\
\hline Alto risco & $\begin{array}{l}\text { Neovasos no disco óptico maior do que } 1 / 3 \text { de sua área. } \\
\text { Hemorragia pré-retiniana ou vítrea acompanhada por neovasos de disco óptico menores } \\
\text { do que } 1 / 3 \text { de área de disco ou neovascularização retiniana maior que } 1 / 2 \text { área de disco. }\end{array}$ \\
\hline
\end{tabular}


dores de DM tipo 2 apresentam RD no momento do diagnóstico de $\mathrm{DM}$, estimando-se que o aparecimento da RD pode preceder em 4 a 7 anos o diagnóstico clínico deste tipo de $\mathrm{DM}^{(23)}$.

Na avaliação inicial do "Wisconsin Epidemiologic Study of Diabetic Retinopathy" (WESRD) um estudo prospectivo observacional de pacientes com DM tipos 1 e 2 desde 1980, a prevalência de RD proliferativa dos pacientes portadores de DM tipo 1 ( $\mathrm{n}=996$ ), foi de $2 \%$ nos pacientes com até dois anos de duração do DM, e de 97,5\% nos pacientes com 15 anos de doença ou mais $^{(13)}$. No mesmo estudo, em pacientes portadores de DM tipo 2 não usuários de insulina ( $\mathrm{n}=674)$, a prevalência de $\mathrm{RD}$ foi de $23 \%$ em pacientes com até 2 anos de DM e 57,3\% em pacientes com 15 anos ou mais de doença. Nos pacientes usuários de insulina, considerados os mesmos tempos de duração de DM $(n=696)$, a prevalência foi de 30 e $84,5 \%$, respectivamente ${ }^{(14)}$. A associação positiva entre duração de $\mathrm{DM}$ e desenvolvimento da RD foi confirmada também na avaliação prospectiva do WESRD em pacientes com DM tipo $1^{(16)}$.

Assim sendo, a duração do DM é um fator a ser levado em consideração em todos os pacientes, independente do controle glicêmico ou do grau de comprometimento oftalmológico.

\section{Controle glicêmico}

A relação entre hiperglicemia e RD tem sido debatida desde a invenção do oftalmoscópio e as primeiras descrições de $\mathrm{RD}$ em $1860^{(24)}$. Com a introdução da medida da glico-hemoglobina na década de 80 , que passou a ser utilizada como uma avaliação acurada do controle metabólico, foi demonstrado ser o controle da glicemia o fator de risco independente mais importante para a $\mathrm{RD}^{(25)}$.

No WESRD, onde uma coorte de 2366 pacientes com DM foi avaliada durante 10 anos, esta observação foi confirmada. A HbAlc inicial foi o mais importante fator de risco para incidência e progressão da RD, mesmo depois de controlado para idade, sexo e duração da doença ${ }^{(26)}$. Em pacientes com DM tipo 1 , o estudo também demonstrou que para cada $1 \%$ de incremento nos valores de $\mathrm{HbA} 1 \mathrm{c}$ o risco de progressão da RD aumenta 1,21 vezes $^{(16)}$.

O "United Kingdom Prospective Diabetes Study" (UKPDS) iniciou em 1977 com o objetivo de avaliar o papel do controle estrito da glicemia e da pressão arterial no desenvolvimento das complicações micro e macrovasculares do DM, acompanhando 4209 pacientes portadores de DM tipo 2 recém diagnosticados. Neste ensaio clínico randomizado, no braço destinado a avaliar o controle glicêmico, os pacientes $(n=3867)$ foram submetidos a tratamento intensivo do DM (sulfoniluréias ou insulina) ou convencional (dieta). Após 10 anos de acompanhamento, demonstrou-se uma redução de $25 \%$ no risco de doença microvascular, no grupo com tratamento intensivo, incluindo hemorragia vítrea e necessidade de fotocoagulação retiniana. Concluiu-se que para cada ponto percentual de redução nos valores de HbAlc (por exemplo, de 9 para $8 \%$ ) houve $35 \%$ de redução no risco de complicações microvasculares ${ }^{(27)}$. Avaliando-se o aparecimento e progressão da RD em 6 anos de acompanhamento nos pacientes do UKPDS, no grupo com controle intensivo, que tinha valores de 6,2 a 7,4\%, o risco relativo (RR) para aparecimento de RD foi de 1,4 , e com valores mais elevados de glico-hemoglobina $(>7,5 \%)$ se observou $R R=2,5$. O risco de progressão da $R D$ (nos pacientes que já apresentavam RD no começo do estudo), considerando-se os mesmos valores de glico-hemoglobina, o RR foi de 4,1 e 8,1, respectivamente ${ }^{(28)}$.

Os resultados de um estudo prospectivo que avaliou o papel do controle glicêmico na prevenção das complicações crônicas relacionadas ao DM em pacientes com DM tipo 2 japoneses também confirmaram o efeito benéfico do controle glicêmico na prevenção da RD. Uma população de 110 pacientes, divididos em coorte de prevenção primária ( $\operatorname{sem} R D, n=55$ ) e prevenção secundária (RD leve), randomizados em grupo de tratamento convencional (insulina de ação intermediária 1 a 2 vezes ao dia) e outro com controle intensivo (insulina regular a cada refeição e insulina intermediária ao deitar), foram acompanhados por 8 anos com oftalmoscopia indireta, retinografia e angiografia a cada 6 meses. No grupo de tratamento intensivo, tanto o aparecimento da RD quanto à progressão de RD foram reduzidos em cerca de $33 \%$ quando comparados com os pacientes sob tratamento convencional ${ }^{(29)}$.

O "Diabetes Control and Complications Trial" (DCCT) foi um ensaio clínico randomizado onde foram avaliados 1441 pacientes portadores de DM tipo 1, sendo 726 sem RD (coorte de prevenção primária) e 715 com RD leve ou moderada (coorte de prevenção secundária) e randomizados em dois grupos: um com controle glicêmico estrito (infusão subcutânea contínua ou múltiplas doses de insulina ao dia) e outro com tratamento convencional (insulina subcutânea 1 a 2 x/dia). Os pacientes foram acompanhados por 4 a 9 anos e realizadas retinografias estereoscópicas a cada 6 meses. O DCCT mostrou que terapia intensiva com insulina reduziu o aparecimento de RD em $42 \%$ quando comparado com o grupo sob terapia convencional. Além disso, a terapia intensiva diminuiu a progressão de RD pré-existente em $32,1 \%$. Esta melhora foi obtida com uma redução média de $10 \%$ nos valores de glico-hemoglobina (de 8 para $7,2 \%)^{(30-31)}$. No prolongamento do estudo, o EDIC (Epidemiology of Diabetes Interventions and Complications) observou-se que, mesmo com controle glicêmico menos rígido, a proporção da incidência de nefropatia e RD, incluindo necessidade de fotocoagulação, edema macular e RD proliferativa continuou menor no grupo que havia sido submetido ao controle intensivo quando comparado com o tratamento convencional ( 72 versus $87 \%)^{(32)}$.

Todos estes estudos demonstram de forma contundente a importância do controle glicêmico em todos os estágios da RD. Deve ser reforçada ao paciente sua importância no sentido de prevenir a RD, especialmente no início do DM.

\section{Nefropatia diabética}

A nefropatia e a RD são complicações microvasculares do $\mathrm{DM}$ que podem estar associadas. A nefropatia diabética acomete de 20 a $30 \%$ dos pacientes portadores de $\mathrm{DM}^{(33)}$. O controle rígido da glicemia reduz a incidência de nefropatia e $\mathrm{RD}$, como demonstrado no American Diabetes Association ${ }^{(34-35)}$, o que reforça a associação entre ambas complicações microvascula- 
res. Vários estudos demonstram a associação de nefropatia diabética e RD. Em estudo de caso-controle em pacientes com DM tipo 1, observou-se que, embora um grande número de pacientes com RD não apresentasse nefropatia (estimada pela microalbuminúria urinária), todos os pacientes com insuficiência renal apresentavam RD proliferativa ${ }^{(17)}$. Outros autores também demonstraram uma maior prevalência de proteinúria maciça em pacientes portadores de RD, principalmente naqueles com RD mais grave ${ }^{(36-38)}$. Esta associação é também observada em pacientes com DM tipo $2^{(39-40)}$.

Esta associação foi também demonstrada em relação à fase inicial da nefropatia. No "Wisconsin Epidemiology Study of Diabetic Retinopathy" (WESDR)(41) após avaliação de 1139 pacientes com DM, se concluiu que a presença de microalbuminúria aumentou em 3 vezes o risco de aparecimento de RD proliferativa em pacientes com DM tipo 1 e não com tipo $2^{(41)}$. De fato, os dados sobre a associação da microalbuminúria com RD em pacientes com DM tipo 2 são conflitantes, com associações positivas $^{(42-43)}$ e sem $\operatorname{associação~}^{(44-45)}$.

Recentemente um estudo de caso-controle realizado no estado do Rio Grande do Sul avaliou fatores associados à RD proliferativa e RD não proliferativa grave em 240 pacientes com DM tipo 2. Foi demonstrada em análise multivariada que a presença de microalbuminúria aumenta em 3,8 vezes a chance do paciente apresentar uma forma grave de $\mathrm{RD}^{(46-47)}$.

\subsection{Fatores de risco não diretamente relacionados ao DM}

\section{Hipertensão arterial sistêmica}

A HAS é duas vezes mais freqüente na população com DM, e parece desempenhar um papel importante na patogênese da $\mathrm{RD}^{(48)}$. Em pacientes portadores de DM existe hiperperfusão do leito capilar em vários tecidos ${ }^{(49)}$. O aumento da pressão arterial aumenta a pressão intraluminar piorando o extravasamento da rede vascular favorecendo a filtração de proteínas plasmáticas através do endotélio e sua deposição na membrana basal do capilar, contribuindo para o dano vascular e a isquemia retiniana aumentando o risco do aparecimento e progressão da $\mathrm{RD}^{(50)}$.

Em pacientes com DM tipo 1, após 14 anos de acompanhamento no WESRD, estimou-se que a hipertensão sistólica e diastólica estava relacionada com a progressão e gravidade da $\mathrm{RD}$ e também com o desenvolvimento de RD proliferativa e edema macular ${ }^{(16)}$.

Em estudo prospectivo de 4 anos envolvendo 153 pacientes portadores de DM tipo 1, com cerca de 23 anos de duração do DM foi estabelecido que valores de pressão diastólica $\geq 70 \mathrm{mmHg}$ já representam risco para progressão da $\mathrm{RD}^{(50)}$. A importância dos níveis de pressão arterial foi confirmada em outro estudo prospectivo em portadores de DM tipo 1 acompanhados por 4 anos, onde se observou que para cada $\geq 10 \mathrm{mmHg}$ de aumento na pressão diastólica, houve $24 \%$ de aumento no risco de progressão da RD. Neste estudo não foi observada relação com pressão arterial sistólica ${ }^{(51)}$.

Já em pacientes com DM tipo 2, um estudo transversal de- monstrou que valores de pressão arterial sistólica $\geq 140 \mathrm{mmHg}$ foram associados a maior prevalência e gravidade da RD, mesmo quando ajustado para duração do DM, controle metabólico e função renal $^{(52) \text {. No UK Prospective Diabetes Study Group }}{ }^{(53)}$, após um seguimento de 9 anos de 1148 pacientes hipertensos com DM tipo 2, os pacientes com controle mais rígido da pressão arterial (média $=144 / 82 \mathrm{mmHg}$ ) quando comparados com pacientes com controle não intensivo (média $=154 / 87 \mathrm{mmHg}$ ) tiveram uma redução de $47 \%$ no risco de perda da acuidade visual. Além disso, o melhor controle pressórico pareceu também ter efeito protetor na ocorrência de edema macular. Estes resultados não foram relacionados ao tipo de tratamento anti-hipertensivo utilizado (captopril ou atenolol) ${ }^{(54)}$. Na mesma população, avaliandose isoladamente a RD, em 6 anos de acompanhamento, os níveis de pressão arterial sistólica estiveram significativamente associados ao desenvolvimento de RD. O RR foi de 1,5 para pressão arterial sistólica entre 125 e $139 \mathrm{mmHg}$, e de 2,8 para pressão arterial sistólica $\geq 140 \mathrm{mmHg}^{(28)}$.

A hipertensão sistólica parece ter um papel preponderante como fator de risco para RD sendo que esta associação com o desenvolvimento desta complicação foi também observada em outras populações. Em estudo transversal com 926 pacientes malaios, na Ásia, a hipertensão sistólica foi o mais importante fator associado à RD em pacientes com DM tipo 1 e tipo 2 diagnosticados antes dos 40 anos de idade ${ }^{(5)}$.

Em estudo caso-controle em pacientes com DM tipo 2 e síndrome plurimetabólica, esta definida pela presença de pelo menos 2 das seguintes características: HAS, dislipidemia, obesidade e microalbuminúria, o fator de risco independente mais importante para presença de RD foi a $\mathrm{HAS}^{(56)}$.

Pode-se concluir que o aumento dos níveis pressóricos é um fator de risco para RD tão importante quanto o controle glicêmico: em pacientes com DM tipo 2 para cada $10 \mathrm{mmHg}$ de redução nos valores de pressão sistólica ocorre uma redução de $13 \%$ nas complicações microvasculares ${ }^{(57-58)}$.

\section{Dislipidemia}

O papel da hipercolesterolemia na RD foi evidenciado em dois estudos longitudinais, o UK Prospective Diabetes Study Group (54) e o "Wisconsin Epidemiologic Study of Diabetic Retinopathy" (WESDR) ${ }^{(59)}$. No WESDR, se observou uma maior presença de exsudatos duros em pacientes com DM tipo 1 e tipo 2 usuários de insulina que tinham colesterol total elevado. Entretanto, a gravidade da RD não foi associada a dislipidemia. Já no ETDRS, um estudo multicêntrico pioneiro na avaliação da eficácia do tratamento precoce a LASER na RD, após acompanhamento de 7 anos de 2709 pacientes com DM tipo 1 e tipo 2, foi demonstrado que um valor de colesterol total $>240 \mathrm{mg} / \mathrm{dL}$ aumentou 2 vezes o risco de desenvolvimento de exudatos duros, e o risco de perda visual em 50\% quando comparado com valores de colesterol total $<200 \mathrm{mg} / \mathrm{dL}$. Resultado similar foi encontrado para LDL colesterol $>160 \mathrm{mg} / \mathrm{dL}$ quando comparado com $\mathrm{LDL}<130 \mathrm{mg} / \mathrm{dL}(\mathrm{RR}=1,97)^{(60)}$.

A influência do colesterol elevado no desenvolvimento da 
RD foi confirmada em um estudo multinacional da OMS sobre doença vascular em diabetes. Em um período de acompanhamento de 8,4 anos, o colesterol sérico foi um fator de risco independente para a RD proliferativa e para perda visual grave ${ }^{(10-11)}$. No mesmo estudo, foram verificados valores mais elevados de triglicerídeos nos pacientes que desenvolveram RD. Entretanto, quando foi realizada análise multivariada esta observação não foi confirmada $^{(11)}$. De fato, não existe associação demonstrada entre hipertrigliceridemia e doença microvascular ${ }^{(61)}$.

\section{Doença cardiovascular}

A doença cardiovascular (doença coronariana, cerebrovascular e vascular periférica) é a principal causa de mortalidade em pacientes com DM tipo 2. Estes pacientes têm de 2 a 4 vezes mais chances de ter eventos cardiovasculares do que pacientes não diabéticos da mesma faixa etária ${ }^{(62)}$.

Muitos dos possíveis fatores de risco relacionados à RD são coincidentes com os fatores de risco para a doença macrovascular do DM, em especial a cardiopatia isquêmica ${ }^{(63)}$. São exemplos a HAS, o fumo e a dislipidemia. De fato, em estudos com longo tempo de acompanhamento, observa-se que a mortalidade por todas as causas é maior nos pacientes portadores de formas mais graves de $\mathrm{RD}^{(24,64)}$.

A incidência de óbito por doença cardiovascular foi avaliada em uma coorte de 408 pacientes portadores de DM tipo 2 com cegueira legal por RD acompanhada por 4 anos. $\mathrm{O}$ óbito por doença cardiovascular foi 5,6 vezes maior nos pacientes portadores de RD incapacitante quando comparados com pacientes sem $\mathrm{DM}(\mathrm{RR}=5,6)^{(65)}$.

Além disso, em estudo prospectivo de 7 anos com 1040 pacientes com DM tipo 2, foi demonstrado através de análise multivariada que o risco de infarto ou morte cardíaca em pacientes com RD proliferativa foi 2 vezes maior do que em pacientes sem esta alteração ${ }^{(63)}$.

A associação entre doença cardíaca aterosclerótica e RD deve ser sempre considerada e, independente da presença de sintomas compatíveis com cardiopatia isquêmica, os pacientes com RD, em especial em estágios avançados, devem ser submetidos a uma avaliação cardiológica.

\section{Obesidade}

A obesidade, um dos componentes da síndrome plurimetabólica $^{(66)}$, representa um estado de resistência insulínica. A possível associação entre resistência insulínica e RD é sugerida pela observação de um estudo retrospectivo envolvendo 534 pacientes com DM tipo 2, onde os pacientes portadores de qualquer estágio de RD apresentaram um maior índice de massa corporal total, bem como uso de doses maiores de insulina ${ }^{(38)}$. Outros fatores determinantes poderiam estar relacionados a esta associação, já que muitos pacientes obesos parecem ter um maior período sem diagnóstico de DM tipo 2 e pior controle metabólico inicial, além de outros fatores, como dislipidemia e maior viscosidade sangüínea ${ }^{(52)}$. Entretanto, recentemente foi observada uma associação entre os componentes da síndrome plurimetabólica e complicações microvas- culares do DM. Pacientes com DM tipo 2 portadores de síndrome plurimetabólica apresentaram mais freqüentemente RD grave $^{(56)}$. Entretanto, o papel da obesidade como fator de risco independente para a RD até o presente momento não está claramente estabelecido.

\section{Gestação e puberdade}

Em pacientes com DM tipo 1, a RD não costuma aparecer antes do início da puberdade, sugerindo sua relação com hormônios sexuais e crescimento ${ }^{(67)}$. Em geral, sugere-se que o rastreamento da doença retiniana não é necessário antes dos 10 anos de idade. Entretanto, recentemente foi observado que a duração pré-puberal do DM parece ser importante no aparecimento de complicações microvasculares ${ }^{(2,68)}$.

A piora das complicações microvasculares no DM durante a gestação, em especial a RD, provavelmente se deve a fatores como instituição de controle glicêmico rígido, presença de fatores de crescimento, HAS e estresse hemodinâmico (sobrecarga) da gestação e parto.

Nas pacientes com DM tipo 1 a progressão da RD durante a gestação é geralmente importante. Estas pacientes têm entre 5 a 20 anos de duração de DM, e uma maior prevalência de alterações retinianas pré-existentes ${ }^{(69)}$. Pacientes com DM tipo 1 gestantes têm risco de cerca de 2,3 vezes maior para progressão da RD, quando comparadas a pacientes com DM tipo 1 não gestantes independente da glico-hemoglobina, duração do DM, idade e pressão $\operatorname{arterial}^{(70)}$.

O papel da gestação como um fator de risco independente para a progressão da RD foi recentemente confirmado. $\mathrm{O}$ "Diabetes Control and Complications Trial Research Group" $(\mathrm{DCCT})^{(71)}$ comparou a progressão de RD em 180 mulheres portadoras de DM tipo 1 que engravidaram com 500 mulheres do mesmo grupo que não engravidaram, em um período de acompanhamento de 6,5 anos. Foi demonstrado um maior risco de piora da RD, tanto nas pacientes que já estavam sob tratamento intensivo $(\mathrm{RR}=1,65)$ quanto nas pacientes que estavam sob tratamento convencional previamente à gestação, embora maior neste último grupo $(\mathrm{R}=2,48)$. Quando o agravamento da RD foi ajustado para as alterações nos valores de glico-hemoglobina, o risco de piora da RD durante a gestação persistiu, estendendo-se por até 1 ano após o parto $(\mathrm{RR}=2,87)$.

Em conclusão, a gestação representa um fator de risco bem definido para a RD e idealmente as pacientes que pretendem engravidar devem ter uma avaliação oftalmológica prévia à concepção e tratamento adequado da RD, se necessário ${ }^{(21)}$. Da mesma forma, devem ser controlados outros fatores de risco bem estabelecidos, como os níveis glicêmico e pressórico. Uma vez iniciado o período gestacional a paciente deve permanecer sob acompanhamento do oftalmologista por até um ano após a concepção.

\subsection{Fatores de risco ambientais}

O hábito de fumar poderia ter efeito deletério sobre a retina com vascularização deficiente dos pacientes portadores de DM pelos seus efeitos isquêmicos: aumento do monóxido de 
carbono, aumento de agregação plaquetária e vasoconstricção. Entretanto, na maior parte dos estudos ${ }^{(52,72-73)}$, incluindo um estudo prospectivo com mais de 10 anos de seguimento ${ }^{(74)}$, o tabagismo não pareceu estar associado à RD.

Em pacientes que participaram do UKPDS, comparando-se em 6 anos de acompanhamento a incidência de RD em fumantes atuais e naqueles que nunca haviam fumado, foi encontrado um efeito protetor do tabagismo $(\mathrm{RR}=0,5)$. Os autores sugerem que esta proteção ocorra por possíveis efeitos farmacológicos da nicotina ou de outros componentes do tabaco ${ }^{(28)}$. É possível que a falta de correlação possa dever-se ao aumento de mortalidade em fumantes, especialmente por doença cardiovascular, o que reduziria as chances deste pacientes desenvolverem $\mathrm{RD}^{(75)}$. Outro estudo sugeriu também um menor risco de aparecimento e progressão da RD em pacientes diabéticos fumantes. Entretanto, esta aparente vantagem desapareceu quando os valores de glicemia foram incluídos na análise ${ }^{(12)}$.

Portanto, o tabagismo não parece conferir risco, ou proteção, para o desenvolvimento de RD.

O consumo de álcool pode estar relacionado a mau controle glicêmico e a aumento dos níveis pressóricos, mas não parece ser um fator de risco para a $\mathrm{RD}^{(72,76)}$, seja para a $\mathrm{RD}$ proliferativa ou para a progressão da RD. Os possíveis efeitos benéficos residiriam no incremento de HDL, redução de agregação plaquetária e dos níveis de fibrinogênio.

O WESRD avaliou a relação entre o risco de microangiopatia e atividade física. Foi estudada a história pregressa e atual da atividade física em pacientes com DM tipo 1. Em mulheres com história de atividade física atual ou na adolescência, a prevalência de RD proliferativa foi menor quando comparadas com mulheres sedentárias, o que não foi observado em homens ${ }^{(77)}$.

Há um consenso de que atividade física pode ter efeito benéfico nas complicações do DM. Entretanto, pacientes com RD em estágios avançados devem evitar atividades extenuantes, que envolvam manobra de Valsalva, golpes ou choques com a cabeça pelo risco de hemorragia vítrea ou descolamento tracional de retina ${ }^{(78-80)}$.

O uso de ácido acetilsalicílico nas doses de até $625 \mathrm{mg} / \mathrm{dia}$ ou de agentes trombolíticos não está associado a aumento de hemorragia vítrea ou influência no aparecimento ou progressão da $\mathrm{RD}^{(81-82)}$. Conclui-se que, respeitadas as contra-indicações usuais, pacientes diabéticos portadores de RD podem utilizar o ácido acetilsalicílico tanto na prevenção primária quanto secundária da doença cardiovascular sem risco adicional para a $\mathrm{RD}^{(81)}$.

\subsection{Fatores de risco oculares}

Pacientes portadores de DM desenvolvem catarata mais precocemente do que pacientes não diabéticos ${ }^{(83)}$. As complicações pós-operatórias da facectomia nestes pacientes são mais freqüentes, provavelmente pela quebra da barreira hemato-aquosa no transoperatório, especialmente se houver RD pré-existente. Independente da técnica cirúrgica utilizada (facectomia extracapsular, facoemulsificação), a facectomia é um fator de risco para a progressão da RD. O risco de piora pode variar de $21 \%{ }^{(84)}$ a $25 \%{ }^{(85)}$. Este risco é maior nos pacientes com maior duração do
DM, pior controle metabólico, em uso de insulina e com RD prévia mais grave ${ }^{(86)}$.

Embora exista a observação de que a presença de glaucoma está associada a menor prevalência e severidade da $\mathrm{RD}^{(87)}$, estes achados não foram comprovados e existem poucos estudos sobre o tema. Em estudo de coorte com 4 anos de acompanhamento, foi demonstrado que pacientes com DM tipo $1 \mathrm{com}$ pressão de perfusão ocular elevada $\{2 / 3$.[PD+(PS-PD)/3]-IOP $\}$ - onde $\mathrm{PD}=$ pressão diastólica, $\mathrm{PS}=$ pressão sistólica, IOP= pressão intra-ocular - apresentaram risco de desenvolvimento de RD de 2,13 vezes para cada $10 \mathrm{mmHg}$ de elevação de perfusão ocular. Tal achado não foi observado em pacientes com DM tipo $2^{(88)}$.

A presença de miopia acima de -2 dioptrias parece ter efeito protetor para o aparecimento e progressão da $\mathrm{RD}^{\left({ }^{86}\right)}$. Tal efeito benéfico foi confirmado em uma coorte de 1210 pacientes com DM tipo 1 e 1780 pacientes com DM tipo $2(\mathrm{RR}=0,4)$, onde a presença de miopia foi um fator protetor para o desenvolvimento da $\mathrm{RD}^{(88)}$.

\section{Fatores de risco genéticos}

O controle dos principais fatores de risco conhecidos para RD não é capaz de prevenir o aparecimento ou progressão da RD na totalidade dos casos, como já foi demonstrado em grandes ensaios clínicos multicêntricos tanto para pacientes com DM tipo 1 quanto para pacientes com DM tipo $2^{(16,34-35)}$. Esta observação sugere a existência de fatores genéticos relacionados à RD. No entanto, não foram realizados até o presente momento, estudos de agregação familiar de RD. Além disto, os estudos que avaliam a associação de RD com etnia ${ }^{(89)}$ e com o sistema HLA ${ }^{(90-92)}$, têm resultados conflitantes.

De uma maneira geral os genes candidatos a serem associados à $\mathrm{RD}$ estão relacionados aos fatores de risco ou a prováveis mecanismos patogênicos relacionados às complicações microvasculares do DM. A avaliação de polimorfismos de genes-candidatos demonstrou uma associação positiva da RD com os seguintes genes: aldose redutase ${ }^{(92-97)}, \mathrm{VEGF}^{(98)}$, glicoproteína de membrana plasmática $\alpha 2 \beta 1$ integrina (receptor plaquetário para colágeno ${ }^{(99)}$ e gene do receptor para produtos finais de glicação avançada (RAGE) ${ }^{(100)}$. Já os polimorfismos dos genes da enzima óxido nítrico sintase endote lial ${ }^{(101)}$, inibidor da ativação do plasminogênio 1 (PAI-1) ${ }^{(102-103)}$, apolipoproteína $\mathrm{E}^{(104)}$, receptor $\beta 3$-adrenérgico ${ }^{(105)}$ e glicoproteína de membrana plaquetária $\alpha \operatorname{IIb} \beta 3$ integrina (receptor plaquetário para fibrinogênio e fator de von Willebrand) não foram associados à $\mathrm{RD}^{(99)}$. Em relação ao polimorfismo do gene da enzima conversora de angiotensina (ECA) a associação com a RD não está estabelecida de forma definitiva, havendo estudos sem associação demonstrada(106-111) e outros com associação positiva ${ }^{(112-113)}$.

Pode-se concluir que vários genes estão relacionados à RD e que o estudo de novos genes candidatos em grupos populacionais diversos e sob a forma de estudos prospectivos poderá permitir a avaliação da influência relativa de cada gene no desenvolvimento e/ou progressão da RD. 


\section{CONCLUSÕES}

A RD continua sendo um grave problema de saúde pública e deve sempre ser entendida como uma doença de abordagem multidisciplinar. Os fatores de risco associados à RD devem ser levados em conta na abordagem de qualquer paciente portador de DM com o objetivo de prevenir o aparecimento e progressão da RD e conseqüente aumento da mortalidade e comprometimento na qualidade de vida.

\section{ABSTRACT}

Diabetic retinopathy (DR) is a chronic complication of diabetes mellitus (DM). After 20 years of diabetes duration Diabetic retinopathy, occurs in $90 \%$ of the type 1 diabetes mellitus patients and in $60 \%$ of the type 2 diabetes mellitus patients, being the main cause of blindness in adults. High glicemia and blood pressure levels, along with the long diabetes mellitus duration, are the main risk factors for diabetes mellitus Diabetic retinopathy. Other factors can also be associated with the loss of vision that occurs in diabetes mellitus. The risk factors can be classified as genetical or non-genetical, the latter including factors related or not to diabetes mellitus, environmental and ocular. Control of the known risk factors and effective treatment are the main basis of the Diabetic retinopathy management. The aim of this review is to provide to the ophthalmologist an up to date information of these factors, with emphasis in the preventive aspects of the visual loss in the patients with diabetes mellitus.

Keywords: Diabetes Mellitus/complications; Diabetic retinopathy/etiology; Risk factors; Glucose tolerance test; Hypertension; Diabetic nephropathies; Low vision

\section{REFERÊNCIAS}

1. American Diabetes Association: clinical practice recommendations 2002. Report of the Expert Committee on the Diagnosis and Classification of Diabetes Mellitus. Diabetes Care 2002;25(Suppl 1):S5-20.

2. Malerbi DA, Franco LJ. Multicenter study of the prevalence of diabetes mellitus and impaired glucose tolerance in the urban brazilian population aged 30-69 yr. Diabetes Care 1992;15:1509-16.

3. American Academy of Ophthalmology Basic and Clinical Science Course.Section 12: Retina and vitreous. 1999-2000. [online] [cited 2003 March 12].Available from: URL: http://149.142.138.19/jseiweb/Education/ educ educact_basicandclinical.htm

4. Brasil. Ministério da Saúde. Estatísticas. [online] [citado 2003 Março 3]. Disponível em URL: http://www.portalweb01.saude.gov.br/saude/aplicacoes/ noticias/noticias_detalhe.cfm?co_seq_noticia $=132$

5. Schellini SA, Moraes Silva MRB, Moraes Silva MA. Diabetes, retinopatia diabética e cegueira. J Bras Med 1994;67:171-4.

6. Gass JD. Retinal vascular diseases. In: Gass JD. Stereoscopic atlas of macular diseases: diagnosis and treatment.4th ed. St. Louis: Mosby; 1997. p.516-27.

7. Cogan DG, Toussant D, Kuwabara T. Retinal vascular patterns IV. Diabetic retinopathy. Arch Opthalmol 1961;66:366-78.

8. Bienkowski J. An overview of the progression of diabetic retinopathy with treatment recommendations. Nurse Pract 1994;19:50-8.

9. Engerman RL. Pathogenesis of diabetic retinopathy. Diabetes 1989;38:1203-6.

10. American Academy of Ophthalmology. Information statement: Eye care for people with diabetes mellitus[onlline][cited 2002 Aug 25]. Available from
URL: http://www.medem.com/search/article display.cfm?path=n:\&mstr=/ ZZZHN8AAHEC.html\&soc=AAO\&srch_typ=-NAU_search.

11. Miki E, Lu M, Lee ET, Keen H, Bennett PH, Russel D. The incidence of visual impairment and its determinants in the WHO Multinational Study of Vascular Disease in Diabetes. Diabetologia 2001;44(Suppl 2):S31-6.

12. Kenn H, Lee ET, Russel D, Miki E, Bennett PH, Lu M, et al. The appearance of retinopathy and progession to proliferative retinopathy: the WHO multinational study of vascular disease in diabetes. Diabetologia 2001;44(Suppl 2):S22-30.

13. Klein R, Klein BE, Moss SE, Davis ME, DeMets DL. The Wisconsin Epidemiologic Study of Diabetic Retinopathy. II. Prevalence and risk factors of diabetic retinopathy when age at diagnosis is less than 30 years. Arch Ophtalmol 1984;102:520-6.

14. Klein R, Klein BE, Moss SE, Davis ME, DeMets DL. The Wisconsin Epidemiologic Study of Diabetic Retinopathy. III. Prevalence and risk factors of diabetic retinopathy when age at diagnosis is 30 or more years. Arch Ophthalmol 1984;102:527-32.

15. Krolewski AS, Warram JH, Rand LI, Christlieb AR, Busick EJ, Kahn CR Risk of proliferative diabetic retinopathy in juvenile-onset type I diabetes: A 40-yr follow-up study. Diabetes Care 1986;9:443-52.

16. Klein R, Klein BEK, Moss SE, Cruickhanks KJ. The Wisconsin epidemiologic study of diabetic retinopathy (WESDR). XVII. The 14-year incidence and progression of diabetic retinopathy and associated risk factors in type 1 diabetes [commented on Ophthalmology 1998;105:1799-80]. Ophthalmology 1998; 105:1801-15.

17. Dyck PJ, Kratz KM, Karnes JR, Litchy WJ, Klein R, Pach JM, et al. The prevalence by staged severity of various types of diabetic neuropathy, retinopathy, and nephropathy in a population-based cohort: The Rochester diabetic neuropathy study [published erratum in Neurology 1993;43:2345]. Neurology 1993;43:817-24.

18. Chen M, Kao C, Chang C, Wu T, FuC, Chen C, et al. Prevalence and risk factors of diabetic retinopathy among noninsulin-dependent diabetic subjects. Am J Ophthalmol 1992;114:723-30.

19. Sparrow JM, McLeod BK, Smith TDW, Birk MK, Rosenthal AR. The prevalence of diabetic retinopathy and maculopathy and their risk factors in the non-insulin-treated diabetic patients of an English town. Eye 1993;7(Pt 1): 158-63.

20. American Diabetes Association. Diabetic Retinopathy. Diabetes Care 2002; 25(Suppl 1):S90-3.

21. Cunha-Vaz J. Lowering the risk of visual impairment and blindness. Diabet Med 1998;15(Suppl 4):S47-50.

22. Krolewski AS, BarzilaYJ, Warram JH, Martin BC, Pfeifer M, Rand LI. Risk of early-onset proliferative retinopathy in IDDM is closely related to cardiovascular autonomic neuropathy. Diabetes 1992;41:430-7.

23. Harris MI, Klein R, Welborn TA, Knuiman MW. Onset of NIDDM occurs at least 4-7 years before clinical diagnosis. Diabetes Care 1992;15:815-9.

24. Klein R. Hyperglycemia and microvascular and macrovascular disease in diabetes. Diabetes Care 1995;18:258-68.

25. Kullberg CE, Arnqvist HJ. Good blood glucose control characterizes patients without retinopathy after long diabetes duration. Diabet Med 1994;12:314-20.

26. Klein R, Klein BE, Moss SE, Cruikshanks KJ. Relationship of hyperglycemia to the long-term incidence and progression of diabetic retinopathy. Arch Intern Med 1994;154:2169-78.

27. UK Prospective Diabetes Study Group (UKPDS) Intensive blood-glucose control with sulphonylureas or insulin compared with conventional treatment and risk of complications in patients with type 2 diabetes (UKPDS 33). [commented on ACP J Club 1999;130:2-3]. Lancet 1998;352:837-53.

28. Stratton IM, Kohner EM, Aldington, SJ, Turner RC, Holman RR, Manley $\mathrm{SE}$, et al. UKPDS 50: risk factors for incidence and progression of retinopathy in type II diabetes over 6 years from diagnosis. Diabetologia 2001;44:156-63.

29. Shichiri M, Kishikawa H, Ohkybo Y, Wake N. Long-term results of the Kumamoto Study on optimal diabetes control in type 2 diabetic patients. Diabetes Care 2000;23(Suppl 2):B21-9.

30. Diabetes Control and Complications Trial. (DCCT) The effect of intensive diabetes treatment on the progression of diabetic retinopathy in insulin-dependent diabetes mellitus. Arch Ophthalmol 1995;113:36-51.

31. Diabetes Control and Complications Trial. (DCCT) The relationship of glycemic exposure $\left(\mathrm{HbA}_{\mathrm{lc}}\right)$ to the risk of development and progression of retinopathy in the diabetes control and complications trial. Diabetes 1995;44:968-83.

32. Diabetes Control and Complications Trial (DCCT). Retinopathy and nephropathy in patients with type 1 diabetes four years after a trial of intensive therapy [published erratum in N Engl J Med 2000;343:1376]. N Engl J Med 2000;342:381-9.

33. American Diabetes Association: clinical practice recommendations 2002 Diabetic nephropathy. Diabetes Care 2002;25(Suppl 1):S85-9. 
34. American Diabetes Association: clinical practice recommendations 2002. Implications of the United Kingdon Prospective Diabetes Study. Diabetes Care 2002;25(Suppl 1):S28-32.

35. American Diabetes Association: clinical practice recommendations 2002. Implications of the Diabetes Control and Complications Trial. Diabetes Care 2002;25(Suppl 1):S25-7.

36. El-Asrar AM, Al-Rubeaan KA, Al-Amro SA, Moharram OA, Kangave D. Retinopathy as a predictor of other diabetic complications. Int Ophthalmol 2001;24:1-11.

37.Olsen BS, Sjolie A, Hougaard P, Johanneseen J, Borch-Johnsen K, Matineli $\mathrm{K}$, et al. A 6-year nationwide cohort study of glicaemic control in young people with type 1 diabetes. Risk markers for the development of retinopathy, nephropathy and neuropathy. Danish Study Group of Diabetes in Childhood. J Diabetes Complications 2000;14:295-300.

38. Schmechel H, Heinrich U. Retinopathy and nephropathy in 772 insulintreated diabetic patients in relation to the type of diabetes. Diabete Metab 1993;19(1Pt2):138-42.

39. Gross JL, Stein ACR, Beck MO, Fuchs SC, Silveiro SP, Azevedo MJ, et al Risk factors for development of proteinuria by type II (non-insulin dependent) diabetic patients. Braz J Med Biol Res 1993;26:1269-78.

40. Klein R, Moss SE, Klein BE. Is gross proteinúria a risk factor for the incidence of proliferative diabetic retinopathy? Ophthalmology 1993; 100:1140-6.

41. Cruickshanks KJ, Ritter LL, Klein R, Moss SE. The association of microalbuminúria with diabetic retinopathy. The Wisconsin Epidemiologic Study of Diabetic Retinopathy. Ophthalmology 1993;100:862-7.

42. Weijers RNM, Merode GG. Retinopathy and microalbuminúria in type 2 diabetes: determinants and time-dependency of the association. Eur J Intern Med 2001;12:28-34.

43. Gall M-A, Hougaard P, Borch-Johnsen K, Parving H-H. Risk factors for development of incipient and overt diabetic nephropathy in patients with noninsulin dependent diabetes mellitus: prospective, observational study. BMJ 1997;314:783-8.

44. Savage S, Estacio RO, Jeffeers B, Schrier RW. Urinaty albumin excretion as a predictor of diabetic retinopathy, neuropathy, and cardiovascular disease in NIDDM. Diabetes Care 1996;19:1243-8.

45. Voutilainen-Kaunisto RM, Teräsvirta ME, Uusitupa MIJ, Niskanen LK. Occurrence and predictors of retinopathy and visual acuity in type 2 diabetic patients and control subjects 10-year follow-up from the diagnosis. J Diabetes Complications 2001;15:24-33.

46. Boelter MC, Moreira JSR, Triches CB, Rodrigues AFP, Copstein E, Lavisnly J, et al. Microalbuminúria as a risk factor for proliferative diabetic retinopathy in type 2 diabetic patients [resumo] In: XXIII Congresso Pan-americano de Oftalmologia, Buenos Aires, Argentina, julho de 2001. p.190.

47. Boelter MC. Fatores associados a retinopatia diabética grave em pacientes com diabete melito tipo 2 [tese]. Porto Alegre: Universidade Federal do Rio Grande do Sul; 2002.

48. Laakso M. Benefits of strict glucose and blood pressure control in type 2 diabetes: lessons from the UK Prospective Diabetes Study. Circulation 1999;99:461-2.

49. Forrester JV, Knott RM. Pathogenesis of diabetic retinopathy and cataract. In: Pickup JC, Williams G. Textbook of diabetes. $2^{\text {nd }}$ ed. Oxford: Blackwell Science; 1997. p.45.

50. Janka HU, Warram JH, Rand LI, Krolewski AS. Risk factor for progression of background retinopathy in long-standing IDDM. Diabetes 1989;39:460-4.

51.Cohen RA, Hennekens CH, Christen WG, Krolewski A, Nathan DM, Peterson $\mathrm{MJ}$, et al. Determinants of retinopathy progression in type 1 diabetes mellitus. Am J Med 1999;107:45-51.

52.Cignarelli M, De Cicco ML, Damato A, Paternostro A, Pagliarini S, Santoro S, et al. High systolic blood pressure increases prevalence and severity of retinopathy in NIDDM patients. Diabetes Care 1992;15:1002-8.

53. UK Prospective Diabetes Study Group. (UKPDS). Tight blood pressure control and risk of macrovascular disease and microvascular complications in type 2 diabetes: UKPDS 38 [comment on BMJ 199812;317:691-2]. BMJ 1998;317:703-13.

54. UK Prospective Diabetes Study Group. (UKPDS). Efficacy of atenolol and captopril in reducing risk of macrovascular and microvascular complications in type 2 diabetes: UKPDS 39 [comment on BMJ 1998;317:691-2]. BMJ 1998;317:713-20.

55. Wan Nazaimoon WM, Letchuman R, Noraini N, Ropilah AR, Zainal M, Ismail IS, et al. Systolic hypertension and duration of diabetes mellitus are important determinants of retinopathy and microalbuminuria in young diabetics. Diabetes Res Clin Pract 1999;46:213-21.
56. Isomaa B, Henricsson M, Almgren P, Tuomi T, Taskinen MR, Groop L. The metabolic syndrome influences the risk of chronic complications in patients type II diabetes. Diabetologia 2001;44:1148-54.

57. Stratton IM, Adler AI, Neil HA, Matthews DR, Manley SE, Cull CA, et al. Association of glycaemia with macrovascular and microvascular complications of type 2 diabetes (UKPDS 35): prospective observational study [comment on BMJ 2000; 321:394-5]. BMJ 2000;321:405-12.

58. Adler AI, Stratton IM, Neil HAW, Yudkin JS, Matthews DR, Cull CA, et al. Association of systolic blood pressure with macrovascular and microvascular complications of type 2 diabetes (UKPDS 36): prospective observational study [commented on BMJ 2000;321:394-5]. BMJ 2000;321:412-9.

59. Klein BE, Moss SE, Klein R, Surawicz TA. The Wisconsin Epidemiologic Study of Diabetic Retinopathy (WESDR). XIII. Relationship of serum cholesterol to retinopathy and hard exudate. Ophthalmology 1991;98:1261-5.

60. Chew EY, Klein ML, Ferris III FL, Remaley NA, Murphy RP, Chantry K, et al. Association of elevated serum lipid levels with retinal hard exudate in diabetic retinopathy. Early Treatment Diabetic Retinopathy Study (ETDRS) Report 22. Arch Ophthalmol 1996;114:1079-84.

61. Kreisberg RA. Diabetic dyslipidemia. Am J Cardiol 1998;82(12A):67U-73.

62. American Diabetes Association. Consensus development conference on the diagnosis of coronary heart disease in people with diabetes: 10-11 February 1998, Miami, Florida. American Diabetes Association. Diabetes Care 1998; 21:1551-9.

63. Miettinen H, Haffner SM, Lehto S, Rönnemaa T, Pyörälà K, Laakso M. Retinopathy predicts coronary heart disease events in NIDDM patients. Diabetes Care 1996;19:1445-8.

64. Klein R, Moss SE, Klein BE, DeMets DL. Relation of ocular and systemic factors to survival in diabetes. Arch Intern Med 1989;149:226-72.

65. Rajala U, Panjunpää H, Koskela P, Keinänen-Kuikaanniemi S. High cardiovascular disease mortality in subjects with visual impairment caused by diabetic retinopathy. Diabetes Care 2000;23:957-61.

66. World Health Organization. Definition and classification of diabetes mellitus and its complications. Geneva: World Health Organization; 1999. 59p.

67. Goldstein DE, Blinder KJ, Ide CH, Wilson RJ, Wiedmeyer HM, Little RR, et al. Glycemic control and development of retinopathy in youth-onset insulin dependent diabetes mellitus. Ophthalmology 1993;100:1125-32;. discussion p.1131-2.

68. Porta M, Sjoelie AK, Chaturverdi N, Stevens L, Rottiers R, Veglio M, et al. Risk factors for progression to proliferative diabetic retinopathy in the EURODIAB Prospective Complications Study. Diabetologia 2001;44:2203-9.

69. Rosenn BM, Miodovnik M. Medical complications of diabetes mellitus in pregnancy. Clin Obstet Gynecol 2000;43:17-31.

70. Klein BE, Moss SE, Klein R. Effect of pregnancy on progression of diabetic retinopathy. Diabetes Care 1990;13:34-40.

71. The Diabetes Control and Complications Trial Research Group. (DCCT). Effect of pregnancy on microvascular complications in the diabetes control and complications trial [commented on Diabetes Care 2001;24:794-6] Diabetes Care 2000;23:1084-91.

72. Moss SE, Klein BE. Association of cigarette smoking with diabetic retinopathy. Diabetes Care 1991;14:119-26.

73. Orchard TJ, Dorman JS, Maser RE, Becker DJ, Ellis D, LaPorte RE, et al. Factors associated with avoidance of severe complications after $25 \mathrm{yr}$ of IDDM. Diabetes Care 1990;13:741-7.

74. Moss SE, Klein BE. Cigarette smoking and ten-year progression of diabetic retinopathy. Ophthalmology 1996;103:1438-42.

75. Solberg Y, Rosner M, Belkin M. The association between cigarette smoking and ocular diseases. Surv Ophthalmol 1998;42:535-47.

76. Moss SE, Klein R, Klein BE. The association of alcohol consumption with the incidence and progression of diabetic retinopathy. Ophthalmology 1994; 101:1962-8.

77. Cruickshanks KJ, Moss SE, Klein R, Klein BE. Physical activity and proliferative retinopathy in people diagnosed with diabetes before age $30 \mathrm{yr}$. Diabetes Care 1992;15:1267-72.

78. Aiello LP, Gardner TW, King GL, Blankenship G, Cavallerano JD, Ferris FL, et al. Diabetic retinopathy. Diabetes Care 1998;21:143-56.

79. Aiello LP, Cahll MT, Wong JS. Systemic considerations in the management of diabetic retinopathy. Am J Ophthalmol 2001;132:760-76.

80. American Diabetes Association: clinical practice recommendations 2002. Diabetes mellittus and exercise. Diabetes Care 2002;25(Suppl 1):S64-8.

81. American Diabetes Association: clinical practice recommendations 2002. Aspirin therapy in diabetes. Diabetes Care 2002;25(Suppl:S78-9.

82. Early Treatment Diabetic Retinopathy Study (ETDRS). Effects or aspirin treatment on diabetic retinopathy. ETDRS report number 8. Ophthalmology 1991;98(5 Suppl):757-65.) 
83. American Academy of Ophthalmology. Basic and Clinical Science Course. Section 11: Lens and cataracts. 1999-2000 [online] [cited 2003 March 12].Available from: URL: http://149.142.138.19/jseiweb/Education/ educ_educact_basicandclinical.htm

84. Zaczek A, Olivestedt G, Zetterström C. Visual outcome after phacoemulsification and IOL implantation in diabetic patients. Br J Ophthalmol 1999; $83: 1036-41$.

85. Mittra RA, Borrillo JL, Dev S, Mieler WF, Koening SB. Retinopathy progression and visual outcomes after phacoemulsification in patients with diabetes mellitus. Arch Ophthalmol 2000;118:912-7.

86. Henricsson M, Heijl A, Janzon L. Diabetic retinopathy before and after cataract surgery [comment on Br J Ophthalmol 1996;80:778-9]. Br J Ophthalmol 1996;80:789-93

87. Frank RN. Etiologic mechanisms in diabetic retinopathy. In: Ryan S, Schachat AP, Murphy RB. Retina. $2^{\text {nd }}$.ed. St. Louis: Mosby; 1994. p.1247.

88. Moss SE, Klein R, Klein BE. Ocular factors in the incidence and progression of diabetic retinopathy. Ophthalmology 1994;101:77-83.

89. Kalter-Leibovici O, Van Dyk DJ, Leibovici L, Loya N, Erman A, Kremer I, et al. Risk factors for development of diabetic nephropathy and retinopathy in Jewish IDDM patients. Diabetes 1991;40:204-10.

90. Agardh A, Gaur LK, Landin-Olson M, Agardh CD, Lernmark A. HLADQB $1 * 0201 / 0302$ is associated with severe retinopathy in patients with IDDM. Diabetologia 1996;39:1313-7.

91. Dornan TL, Ting A, McPherson CK, Peckar CO, Mann JI, Turnes RC, et al. Genetic susceptibility to the development of retinopathy in insulin-dependent diabetics. Diabetes 1982;31:226-31.

92.Wong TY, Cruickshank KJ, Klein R, Klein BE, Moss SE, Palta M, et al. HLA-DR3 and DR-4 and their relation to the incidence and progression of diabetic retinopathy. Ophthalmology 2002;109:275-81.

93. Demaine A, Cross D, Millward A. Polymorphisms of the aldose reductase gene and susceptibility to retinopathy in type 1 diabetes mellitus. Invest Ophthalmol Vis Sci 2000;41:4064-8.

94. Fujisawa T, Ikegami H, Kawagucchi Y, Yamato E, Nakagawa Y, Shen G, et al. Length rather than a specific allele of dinucleotide repeat in the 5' upstream region of the aldose reductase gene is associated with diabetic retinopathy. Diabet Med 1999;16:1044-7.

95. Ko BC, Lam K, Wat N, Chung S. An (A-C) dinucleotide repeat polymorphic marker at the 5' end of the aldose reductase gene is associated with early-onset diabetic retinopathy in NIDDM patients. Diabetes 1995;44:727-32.

96. Kao YL, Donaghue K, Chan A, Knight J, Silink M An aldose reductase intragenic polyorphism associated with diabetic retinopathy. Diabetes Res Clin Pract 1999;46:155-60.

97. Shimizu H, Ohtani KI, Tsuchiya T, Sato N, Tanaka Y, Takahashi H, et al. Aldose reductase mRNA expression is associated with rapid development of diabetic microangiopathy in japanese type 2 diabetic (T2DM) patients. Diabetes Nutrit Metab 2000;13:75-9.

98. Awata T, Inoue K, Kurihara S, Ohkubo T, Watanabe M, Inukai K, et al. A common polymorphism in the 5'-intranslated region of the VEGF gene is associated with diabetic retinopathy in type 2 diabetes. Diabetes 2002; 51:1635-9.

99. Matsubara Y, Murata M, Maruyama T, Handa M, Yagamata N, Watanabe G, et al. Association between diabetic retinopathy and genetic variations in alpha2beta1 integrin, a platelet receptor for collagenn. Blood 2000;95:1560-4.
100. Hudson BI, Stickland MH, Futers S, Grant PJ. Effects of novel Polymorphis$\mathrm{ms}$ in the RAGE gene on transcriptional regulation and their association with diabetic retinopathy. Diabetes 2001;50:1505-11.

101. Taverna MJ, Sola A, Guyot-Argenton C, Pacher N, Bruzzo F, Chevalier A, Slama G, Reach G, Selam JL. eNOS4 polymorphism of the endothelial nitric oxide synthase predicts risk for severe diabetic retinopathy. Diabet Med 2002;19:240-5.

102. Liu ZH, Guan TJ, Chen ZH, Li LS. Glucose transporter (GLUT-1) allele (Xba-1) associated with nephropathy an non-insulin-dependent diabetes mellitus. Kidney Int 1999;55:1843-8.

103. Tarnow L, Grarup N, Hansen T, Parving H, Pedersen O. Diabetic microvascular complications in the GLUT-1 and PC-1 genes regulating glucose metabolism in Caucasian type 1 diabetic patients. Nephrol Dial Transplant 2001;16:1653-6.

104. Tarnow L, Stehouver CD, Emeis JJ, Poirier O, Cambien F, Hansen BV, et al. Plasminogen activator inibitor-1 and apolipoprotein $\mathrm{E}$ gene polymorphisms and diabetic angiopathy. Nephrol Dial Transplant 2000;15:625-30.

105. Tarnow L, Urhammer SA, Mottalu B, Hansen B, Pedersen O, Parving H. The Trp64Arg amino acid polymorphism of the $\beta_{3}$-adrenergic receptor gene does not contribute to the genetic susceptibility of diabetic microvascular complications in caucasian type 1 diabetic patients. Nephrol Dial Transplant 1999;14:895-7.

106. Fujisawa T, Ikegami H, Kawaguchi Y, Hamada Y, Ueda H, Shintani M, et al. Meta-analysis of association of insertion/deletion polymorphism of angiotensin I-converting enzyme gene myocardial diabetic nephropathy and retinopathy. Diabetologia 1998; 41:47-53.

107. Kennon, B Petrie JR, Small M, Conell JMC. Angiotensin-converting enzyme gene and diabetes mellitus. Diabet Med 1999;16:448-58.

108. Marre M, Bernadet P, Gallois Y, Savagner F, Guyene T, Hallab M, et al. Relationships between angiotensin I converting enzyme gene polymorphism, plasma levels, and diabetic retinal and renal complications. Diabetes 1994; 43:384-8.

109. Grzeszcak W, Zychma MJ, Lacka B, Zukowska-Szczechowska E. Angiotensin I-converting enzyme gene polymorphisms: relationship to nephropathy in patients with non-insulin dependent diabetes mellitus. J Am Soc Nephrol 1998;9:1664-9.

110. Araz M, Yilmaz N, Güngör K, Okan V, Kepekci Y, Sükrü Aynacioglu A. Angiotensin-converting enzyme gene polymorphism and microvascular complications in turkish type 2 diabetic patients. Diabetes Res Clin Pract 2001; 54:95-104.

111. Fujisawa T, Ikegami H, Shen Q, Yamato L, Takekawa T, Nakagawa T, et al. Angiotensin I-converting enzyme gene polymorphism is associated with myocardial infarction, but not with retinopathy or nephropathy in NIDDM [comment on Diabetes Care 1996;19:543-4]. Diabetes Care 1995;18:983-5.

112. Rabensteiner D, Abrahamian H, Irsigler K, Hermann KM, Kiener HP, Mayer $\mathrm{G}$, et al. ACE gene polymorphism and proliferative retinopathy in type 1 diabetes: results of a case-control study. Diabetes Care 1999;22:1530-5.

113. Matsumoto A, Iwashima Y, Abiko A, Morikawa A, Sekiguchi M, Eto M, et al. Detection of the association between a deletion polymorphism in the gene encondig angiotensin I-converting enzyme and advanced diabetic retinopathy. Diabetes Res Clin Pract 2000;50:195-202.

Nos artigos enviados para publicação, o nome dos autores

e suas afiliações devem estar completos. Isso facilitará a indexação e os links com as bases de dados e o CV Lates. 